\title{
Relationship between platelet-based models and the prognosis of patients with malignant hepatic tumors
}

\author{
CHEN-LIANG HU ${ }^{1 *}$, QIAN-CHENG DU ${ }^{2 *}$, ZHI-XIN WANG ${ }^{1}$, MING-QUAN PANG $^{1}$, \\ YAN-YAN WANG ${ }^{3}$, YING-YU LI ${ }^{4}$, YING ZHOU ${ }^{1}$, HAI-JIU WANG ${ }^{1}$ and HAI-NING FAN ${ }^{1}$ \\ ${ }^{1}$ Department of Hepatopancreatobiliary Surgery, The Affiliated Hospital of Qinghai University and Qinghai Province \\ Key Laboratory of Hydatid Disease Research, Qinghai University, Xining, Qinghai 81000; ${ }^{2}$ Department of General Surgery, \\ Shanghai Fourth People's Hospital Affiliated to Tongji University School of Medicine, Shanghai 200081; \\ ${ }^{3}$ Department of Hematology, Affiliated Fuyang Hospital of Anhui Medical University, Fuyang, Anhui 236000; \\ ${ }^{4}$ Department of Medical Record Room, The Affiliated Hospital of Qinghai University, \\ Qinghai University, Xining, Qinghai 81000, P.R. China
}

Received April 22, 2019; Accepted November 29, 2019

DOI: $10.3892 / 01.2020 .11317$

\begin{abstract}
Platelets (PLTs) are involved in tumor growth, metabolism and vascular activation. PLT-based models have been reported to have significant value on the recurrence of malignant hepatic tumors. The present study aimed to investigate the effect of PLT count and 18 PLT-based models on the prognosis of patients with malignant hepatic tumors. The clinical data from 189 patients with malignant hepatic tumors were retrospectively analyzed and used to calculate the scores of the 18 PLT-based models. Receiver operating characteristic curve was used to determine the suitable cut-off values of mortality and recurrence in patients with malignant hepatic tumors. The overall survival and cumulative recurrence rates of patients were calculated using Kaplan-Meier survival curves and the difference was analyzed using log-rank test. Multivariate analysis was performed to determine the independent risk factors of recurrence-free survival and overall survival. In the present study, 11 models were considered as predictors of mortality $(\mathrm{P}<0.05)$ and six models were considered as predictors of recurrence $(\mathrm{P}<0.05)$. The results from multivariate analysis demonstrated that vascular cancer embolus, uric acid $>231 \mu \mathrm{mol} / 1$, hemoglobin $>144 \mathrm{~g} / 1$ and the Lok index model $>0.695$ were considered as independent risk
\end{abstract}

Correspondence to: Professor Hai-Jiu Wang or Professor Hai-Ning Fan, Department of Hepatopancreatobiliary Surgery, The Affiliated Hospital of Qinghai University and Qinghai Province Key Laboratory of Hydatid Disease Research, Qinghai University, 29 Tongren Road, Xining, Qinghai 81000, P.R. China

E-mail: 18897052024@163.com

E-mail: fanhaining@medmail.com.cn

${ }^{*}$ Contributed equally

Key words: platelet, model, prognosis, hepatic tumor, recurrence factors of mortality $(\mathrm{P}<0.05)$. Furthermore, vascular cancer embolus, PLT to lymphocyte ratio (PLR) $>175$ and fibrosis-4 (FIB-4) $>4.82$ were independent factors of recurrence $(\mathrm{P}<0.05)$. In addition, the results from this study indicated that the Lok-index could be considered as a predictor of the overall survival rate. In conclusion, the FIB-4 and PLR model may be valuable for predicting the recurrence-free rate of patients with malignant hepatic tumors.

\section{Introduction}

Liver cancer is the fourth leading cause of cancer-associated mortality worldwide, with $\sim 4.7 \%(841,080 / 18,078,957)$ new cases and accounted for $8.2 \%(781,631 / 9,555,027)$ of all types of cancer-associated deaths worldwide in 2018 (1). The main risk factors for the occurrence of hepatocellular carcinoma include alcoholic cirrhosis, fungal aflatoxin infection, and hepatitis B and C viral infection (2). Although the diagnosis and treatment of hepatic carcinoma have improved recently, the majority of patients are diagnosed or present with symptoms at middle and advanced stages and have a low survival rate of $5-6 \%$ (3), which is due to the discreet clinical symptoms, subtle onset, invasive growth and high malignancy in the early stage.

Platelets (PLTs) are the smallest type of blood cell and PLT counts are typically between 100 and $300 \times 10^{9} / 1$. PLTs release numerous cytokines that participate in the inflammatory response, such as PLT-derived growth factors and transforming growth factor $\beta$. PLTs also transport these substances to specific locations, therefore PLTs serve important roles in numerous functions, including angiogenesis, wound healing and liver regeneration. Alterations in the number and function of PLTs may lead to numerous physiological and pathological changes, such as in the inflammatory response and thrombosis (4), and may thus result in serious complications and diseases such as venous thromboembolism (5). The majority of patients with liver cancer present with cirrhosis, which is generally caused by chronic hepatitis (6). The decompensated 
stage of cirrhosis is characterized by portal hypertension, hypersplenism and low PLT count $(7,8)$. During the development of liver cancer, cancer cells can cause an imbalance in the blood coagulation system via the overproduction of blood coagulation factors. Furthermore, this imbalance can promote excessive PLT activation $(9,10)$. The activated PLTs serve as a procoagulant surface, inducing cancer-associated coagulation, and the activated PLTs may also be recruited to surround tumor cells, assisting immune evasion of tumor cells and thus cytolysis by killer cells $(5,11)$. In addition, PLTs are recruited to surround tumor cells, to protect them from the body's immune system and to promote their proliferation and metastasis $(12,13)$. Furthermore, PLTs may release growth factors that stimulates cellular growth, proliferation, healing, and cellular differentiation, such as transforming growth factor- $\beta$ and fibroblast growth factor, which increases invasive capacity and proliferation of cancer cells $(5,14,15)$. In addition, PLTs and numerous noninvasive models, such as AAR, AST to PLT ratio index (APRI) (16), fibrosis-4 (FIB-4) (17), Pohl score (18), FibroQ (19) and Lok index (20), have been reported to predict liver fibrosis and are therefore considered diagnostic indicators of cirrhosis (20-23). Previous studies demonstrated that PLTs represent independent factors of cancer recurrence and prognosis (24-26). The present study hypothesized therefore that PLT-based models may be considered as crucial factors for the prognosis of patients with malignant hepatic tumors.

Although recent studies have demonstrated that abnormal PLT counts are associated with a poor prognosis in patients with cancer, this association remains controversial $(27,28)$. In addition, previous studies have reported a correlation between PLT-based models and the recurrence and overall survival rate in patients with malignant hepatic tumors (15,29-32). In the present study, 18 PLT-based models were used to predict the overall survival and recurrence-free survival in patients with malignant hepatic tumors.

Malignant hepatic tumors are currently diagnosed using imaging techniques, including abdominal ultrasound, abdominal computed tomography (CT) and abdominal magnetic resonance imaging (MRI) (33-36). Although ultrasound is a simple method widely used for the screening of liver cancer, $\mathrm{CT}$ and MRI are the primary methods for diagnosing hepatic carcinoma. Furthermore, there are only a few available biomarkers for diagnosis of liver cancer, such as a-fetoprotein (AFP) (37); however, analysis of pathological tissue biopsy remains the most efficient diagnostic.

Numerous methods for the treatment of malignant hepatic tumors exist, including surgical and non-surgical treatments. Liver transplant is an effective treatment for patients with complex or end-stage lesions (38). Non-surgical treatments comprise radiofrequency ablation, microwave ablation (liver tumor $<4 \mathrm{~cm}$ ), radiotherapy and systemic chemotherapy (39-41). Systemic chemotherapy, including targeted therapy by sorafenib, provided to patients with distant metastases and unresectable lesions (Barcelona Clinic Liver Cancer stage C/D) (41), resulted in an increase in overall survival rate when combined with other chemotherapeutics. Immunotherapy-based regimens and novel chemotherapeutic agents may improve outcomes for patients with HCC. All therapies and treatments will have certain contraindications in specific patients. Therefore, personalized regimens for patients are required necessary to improve outcomes in patients with HCC (39).

\section{Patients and methods}

Patients. The clinical data of 189 patients with malignant hepatic tumors who received surgery and non-surgical treatment during January 2011 and March 2018 at the Affiliated Hospital of Qinghai University were collected. Among these patients, 145 were male and 44 were female, with a mean age of $56 \pm 10$ years (range, 16-82 years). Patients who received surgery were pathologically diagnosed with malignant hepatic tumors whereas those who did not receive surgery were diagnosed with using imaging based techniques. This study was approved by the Institutional Research Ethics Board of Qinghai University Affiliated Hospital and conformed to the Declaration of Helsinki. Written informed consent was provided by all patients.

Study design. The clinical data of patients included sex, age, hepatitis B virus (HBV) infection status, presence of cirrhosis or ascites, the Child-Pugh score (42) were collected (42), preoperative relevant laboratory indicators (blood routine examination, biochemical test and coagulation function), surgical records and tumor imaging characteristics. Patients were selected according to the following inclusion criteria: i) Diagnosis of hepatic malignant tumor made by histopathological analysis or imaging; ii) diagnosed and treated at the Department of Hepatopancreatobiliary Surgery, The Affiliated Hospital of Qinghai University; iii) patients had no history of surgery prior to hospitalization; iv) no adjuvant chemotherapy or radiotherapy was administered prior to or following treatment, including hepatectomy or non-surgical treatment; v) patients had no systemic inflammatory response syndrome; vi) patients had no coexistent hematologic diseases; and vii) patients had no history of blood transfusion for three months prior to treatment, including hepatectomy and non-surgical treatment. Patients who died from non-cancerous-associated causes or whose data were incomplete were excluded.

PLT-based model. The scoring models adopted in this study were as follows: Pohl score, aspartate aminotransferase/alanine aminotransferase ratio-platelet count score (AARP) (43), asthma predictive index (API) (44), care dependency scale (CDS) (22), AST to PLT ratio index (APRI), fibrosis-4 (FIB-4), FibroQ, Göteborg University Cirrhosis Index (GUCI) (45), King's score (46), $\gamma$-glutamyl transpeptidase to PLT ratio (GPR) (47), S-index (48), Forns index (49), Platelet count/age/ALP/AFP/AST index(PAPAS) (50), Aspartate- aminotransferase/platelet count/GPR/AFP index (APGA) (51), fibrosis index based on the three factors (Lok index), P2/MS (52), periodontal screening and recording (53) and PLT to lymphocyte ratio (PLR) (54). The algorithms of the inclusive 17 scoring systems, including Pohl score, AARP, API, CDS, APRI, FIB-4, FibroQ, Lok-index, GUCI, APGA, PAPAS, King's score, GPR, S-index, PSR, P2/MS and Forns index, have been previously used in transhepatic arterial chemotherapy and embolization therapy (32). 
Follow-up. The beginning of the follow-up corresponds to the date of the initial diagnosis. In the present study, the cut-off date was either the time of the last follow-up (March 2018) or the death of the patient. Event outcomes included mortality and survival. The follow-up included assessment of survival and recurrence. Recurrence was assessed based on the clinical characteristics, imaging examination, expression levels of tumor markers, including AFP, and pathological diagnosis of hepatic malignancy by analyzing changes in tumor cell morphology, tissue structure and growth pattern. Malignant hepatic tumors are classified into primary hepatic carcinoma (PHC) and secondary hepatic carcinoma. PHC includes hepatocellular carcinoma (HCC), intrahepatic cholangiocarcinoma and other rare subtypes (1). In addition, recurrence-free survival was evaluated from the beginning of treatment until the detection of local or distant recurrence. Overall survival was estimated from the beginning of treatment until death (55).

Statistical analysis. Statistical analysis was performed using SPSS software version 23.0 (IBM Corp.). Figures were generated using GraphPad Prism 5.01 (GraphPad Software, Inc.). Student's test and $\chi^{2}$ test were used to compare the continuous and categorical variables, respectively. The rank sum test was used for comparison of continuous data that did follow a normal distribution. The receiver operating characteristic curve (ROC) was calculated to analyze the predictive ability of the 18 scoring systems for the overall survival and recurrence-free survival of patients with malignant hepatic tumors and was used to determine the optimal cut-off point (with the highest specificity and sensitivity sum) of each variable. All scoring systems found to be significant $(\mathrm{P}<0.05)$ using ROC curve analysis were subsequently further assessed using Kaplan-Meier survival analysis. Kaplan-Meier analysis was used to estimate the cumulative survival and recurrence rates and a log-rank test was used to analyze the differences between two groups. $\mathrm{P}<0.05$ was considered to indicate a statistically significant difference.

All variables that were demonstrated to be significant $(\mathrm{P}<0.05)$ were then analyzed by multivariate analysis with Cox proportional hazard regression models. The continuous variables of normal distribution were presented as means \pm standard deviation, otherwise it was presented as median (minimum-maximum).

\section{Results}

Patient characteristics. The present study included 93 patients who were infected with HBV, 80 patients who presented with cirrhosis, 96 patients who had received surgical treatment and 93 patients who had been treated with non-surgical therapy such as microwave ablation. According to the Kaplan-Meier curve, the median survival time of 189 patients was 46 months. Furthermore, after an average follow-up period of 24.06 months, $60.8 \%(115 / 189)$ of patients survived, $39.2 \%$ (74/189) of patients experienced recurrence and $39.2 \%$ (74/189) of patients died in Table I. The clinicopathological characteristics, serological tests, tumor characteristics and scoring models of all patients, classified according to the survival status, are presented in Table I. Significant differences were observed between the two groups (survival and mortality) in the number of tumor lesions, level of total protein, uric acid, recurrence, Pohl score, CDS, APRI, King's score, Forn index, Lok index, FibroQ, PAPAS, FIB-4, GUCI, GPR, APGA and PLR $(\mathrm{P}<0.05)$. However, sex, age, $\mathrm{HBsAg}$, ascites, cirrhosis, Child-Pugh score, diameter of spleen (mm), hypertension, polycythemia, the method of treatment (surgical treatment vs. non-surgical treatment), tumor size, vascular cancer embolus, ALB, CEA, ALT, AST, ALP, GGT, AFP level, PLT, PT, INR, neutrophil (\%), mononuclear cell (\%), AARP, API, P2/MS, PSR and S-index were not statistically different between the two groups $(\mathrm{P}>0.05)$.

The clinicopathological characteristics, serological tests, tumor characteristics and scoring models of all patients, classified according to the recurrence status, are presented in Table II. Child-Pugh classification, percentage of lymphocyte, percentage of neutrophil and cholesterol level exhibited significant differences between the two groups $(\mathrm{P}<0.05)$. The method of treatment (surgical treatment vs. non-surgical treatment), tumor size, lesion number, presence of cirrhosis and AFP level were not statistically different between the two groups $(\mathrm{P}>0.05)$.

Optimal cut-off point. According to the results from ROC curves (Fig. 1), the cut-off value of PLT for the prediction of survival was $113 \times 10^{9} \mathrm{~g} / \mathrm{l}$ because of the maximal sum of sensitivity plus specificity. Furthermore, AFP [area under the curve (AUC), 0.610; 95\% confidence interval (CI), 0.527-0.671; $\mathrm{P}=0.0133$ ], uric acid value (AUC, 0.614; 95\% CI, 0.541-0.684; $\mathrm{P}=0.0057$ ), total albumin (AUC, 0.585; 95\% CI, 0.511-0.656; $\mathrm{P}=0.0429$ ), APGA (AUC, 0.613; 95\% CI, 0.540-0.683; $\mathrm{P}=0.0061$ ), APRI (AUC, 0.606; 95\% CI, 0.533-0.676; $\mathrm{P}=0.0121), \mathrm{CDS}$ (AUC, 0.610; 95\% CI, 0.536-0.680; $\mathrm{P}=0.0078$ ), FIB-4 (AUC, 0.615; 95\% CI, 0.541-0.685; P=0.0067), FibroQ (AUC, 0.610; 95\% CI, 0.537-0.680; P=0.0099), Forns-index (AUC, 0.595; 95\% CI, 0.522-0.666; P=0.0258), GPR (AUC, 0.591; 95\% CI, 0.518-0.662; P=0.0297), GUCI (AUC, 0.606; 95\% CI, 0.533-0.676; $\mathrm{P}=0.0120$ ), King's score (AUC, 0.612; 95\% CI, 0.539-0.682; $\mathrm{P}=0.0077$ ), Lok-index (AUC, 0.597; 95\% CI, 0.523-0.667; P=0.0254), PAPAS (AUC, 0.608; 95\% CI, 0.535-0.678; $\mathrm{P}=0.0090$ ) and PLR (AUC, 0.662; 95\% CI, $0.590-0.729 ; \mathrm{P}=0.001)$ could significantly predict patient survival (Table III). Among these indicators, the AUC of PLR was the largest (AUC=0.662), indicating that the predictive potential of PLR for predicting outcomes was improved compared with the other models.

Regarding the ability of these indicators to predict recurrence of malignant hepatic tumors in patients, the percentage of lymphocytes (AUC, 0.597; 95\% CI, 0.523-0.667; P=0.0191), level of uric acid (AUC, 0.583; 95\% CI, 0.509-0.654; $\mathrm{P}=0.0482$ ), percentage of neutrophils (AUC, 0.615; 95\% CI, 0.542-0.685; $\mathrm{P}=0.0048$ ) and PLR (AUC, 0.640; 95\% CI, 0.567-0.708; $\mathrm{P}=0.0007)$ were significant predictors of recurrence (Table IV). APGA (AUC, 0.542; 95\% CI, 0.443-0.590; P=0.6892), APRI (AUC, 0.517; 95\% CI, 0.443-0.590; P=0.6892), FibroQ (AUC, $0.525 ; 95 \% \mathrm{CI}, 0.451-0.598 ; \mathrm{P}=0.5608$ ), Forns index (AUC, 0.505; 95\% CI, 0.431-0.578; $\mathrm{P}=0.9105$ ), GPR (AUC, 0.500; 95\% CI, 0.427-0.574; $\mathrm{P}=0.9968$ ), GUCI (AUC, 0.511; 95\% CI, 0.437-0.584; $\mathrm{P}=0.7994$ ) and King's score (AUC, 0.508, 95\% CI, 0.435-0.582; $\mathrm{P}=0.8430$ ) were not statistically significant. Among these scoring systems, only the PLR (AUC, 0.640; 95\% CI, 0.567-0.708; $\mathrm{P}=0.0007)$ was a significant predictor of recurrence. 
Table I. Characteristics of patients with malignant hepatic tumors categorized according to survival status.

\begin{tabular}{|c|c|c|c|}
\hline Parameter & Survival cases & Mortality cases & P-value \\
\hline Sex, male/female, $\mathrm{n}$ & $83 / 32$ & $62 / 12$ & 0.065 \\
\hline Age, years & $54 \pm 11$ & $57 \pm 9$ & 0.236 \\
\hline HBsAg, negative/positive, $n$ & $62 / 53$ & $34 / 40$ & 0.285 \\
\hline Ascites, no/yes, $\mathrm{n}$ & $107 / 8$ & $68 / 6$ & 0.768 \\
\hline Cirrhosis, no/yes, $\mathrm{n}$ & $71 / 44$ & $38 / 36$ & 0.158 \\
\hline Child-Pugh classification, A/B, n & $98 / 17$ & $65 / 9$ & 0.611 \\
\hline Diameter of spleen, mm (range) & $106.9(72.8-194.1)$ & $110.1(75.3-175.3)$ & 0.196 \\
\hline Hypertension, no/yes, n & $107 / 8$ & $65 / 9$ & 0.222 \\
\hline Polycythemia, no/yes, $n$ & $111 / 4$ & $69 / 5$ & 0.317 \\
\hline Diabetes, no/yes, $\mathrm{n}$ & $110 / 5$ & $72 / 2$ & 0.707 \\
\hline Non-surgical/surgical treatment, $\mathrm{n}$ & $53 / 62$ & $40 / 34$ & 0.285 \\
\hline Tumor size, $<5 / \geq 5 \mathrm{~cm}, \mathrm{n}$ & $36 / 79$ & $24 / 50$ & 0.871 \\
\hline Number of tumor lesions, single/multiple, $\mathrm{n}$ & $90 / 25$ & $45 / 29$ & 0.010 \\
\hline Vascular cancer embolus, no/yes, $\mathrm{n}$ & $103 / 12$ & $59 / 15$ & 0.059 \\
\hline $\mathrm{ALB}, \mathrm{U} / 1$ & $37.81 \pm 5.80$ & $37.51 \pm 5.348$ & 0.725 \\
\hline CEA, ng/ml (range) & $2.18(0.50-735.44)$ & $2.37(0.51-954.63)$ & 0.610 \\
\hline ALT, U/l (range) & $41(11-661)$ & $42.35(7-212)$ & 0.542 \\
\hline AST, U/l (range) & $47(13-661)$ & $53(16-228)$ & 0.065 \\
\hline ALP, U/1 (range) & $120(20-908)$ & $143(62-1854)$ & 0.110 \\
\hline GGT, U/l (range) & $92(5-1257)$ & $119(12-739)$ & 0.210 \\
\hline $\mathrm{AFP},<200 / \geq 200 \mathrm{ng} / \mathrm{ml}, \mathrm{n}$ & $71 / 44$ & $43 / 31$ & 0.619 \\
\hline Total protein, g/l (range) & $68(31-97)$ & $70(50-92)$ & 0.049 \\
\hline PLT, $\times 10^{9} / 1$ (range) & $139(38-537)$ & $129(37-366)$ & 0.089 \\
\hline PT (range) & $12.8(8.9-19.9)$ & $12.8(10-20.2)$ & 0.534 \\
\hline INR (range) & $1.06(0.75-1.63)$ & $1.065(0.84-1.74)$ & 0.546 \\
\hline Mononuclear cell, \% (range) & $6.9(1.2-23.51)$ & $7.55(3.24-18.81)$ & 0.056 \\
\hline Neutrophil, \% (range) & $61.53 \pm 12.19$ & $58.17 \pm 14.34$ & 0.086 \\
\hline Uric acid, $\mu \mathrm{mol} / 1$ (range) & $270(11-538)$ & $305(133-549)$ & 0.008 \\
\hline AARP, negative/positive & $21 / 94$ & $12 / 62$ & 0.718 \\
\hline Pohl score, negative/positive & $86 / 29$ & $40 / 34$ & 0.003 \\
\hline API (range) & $6(0-10)$ & $7(1-10)$ & 0.085 \\
\hline CDS (range) & $6(2-10)$ & $6(2-9)$ & 0.011 \\
\hline APRI (range) & $0.87(0.11-16.47)$ & $1.25(0.16-10.17)$ & 0.014 \\
\hline King's score (range) & $20.46(2.03-30.5 .1)$ & $31.85(3.68-239.28)$ & 0.009 \\
\hline Lok index (range) & $0.50(0.02-0.99)$ & $0.70(0.06-0.99)$ & 0.025 \\
\hline $\mathrm{P} 2 / \mathrm{MS}$ (range) & $56.65(1.05-625.01)$ & $38.56(1.98-1,684.78)$ & 0.094 \\
\hline PAPAS (range) & $2.92(1.29-5.73)$ & $3.22(1.17-8.97)$ & 0.012 \\
\hline PSR (range) & $1.34(0.28-4.99)$ & $1.13(0.35-4.75)$ & 0.070 \\
\hline S-index (range) & $0.44(0.02-10.9)$ & $0.68(0.05-4.84)$ & 0.067 \\
\hline FIB-4 (range) & $2.63(0.49-24.5)$ & $3.29(0.78-26.59)$ & 0.008 \\
\hline FibroQ (range) & $4.24(0.91-45.83)$ & $5.82(1.16-45.12)$ & 0.011 \\
\hline GUCI (range) & $35.51(4.72-663.26)$ & $51.56(6.23-498.51)$ & 0.014 \\
\hline GPR (range) & $0.58(0.03-13.66)$ & $0.94(0.05-6.1)$ & 0.034 \\
\hline APGA (range) & $17.01(4.04-65.83)$ & 20.87 (4.39-68.33) & 0.009 \\
\hline Forn index (range) & $9.71(3.34-13.75)$ & $10.19(5.91-14.28)$ & 0.027 \\
\hline PLR (range) & $94.60(18.67-589.29)$ & $128.06(31.60-588.64)$ & $<0.001$ \\
\hline Recurrence, no/yes, n & $99 / 16$ & $16 / 58$ & $<0.001$ \\
\hline
\end{tabular}

ALB, albumin; CEA, carcinoembryonic antigen; ALT, alanine aminotransferase; AST, aspartate aminotransferase; ALP, alkaline phosphatase; GGT, $\gamma$-glutamyl transpeptidase; AFP, $\alpha$-fetoprotein; PLT, platelet; API, age-PLT index; CDS, care dependency scale; APRI, AST to PLT ratio index; PSR, PLT count/spleen diameter ratio; FIB-4, fibrosis-4; GUCI, Göteborg University Cirrhosis Index; GPR, $\gamma$-glutamyl transpeptidase to PLT ratio; APGA, Aspartate aminotransferase/platelet count/ $\gamma$-glutamyl transpeptidase/alpha fetoprotein index; PLR, PLT to lymphocyte ratio; PT, Prothrombin time; INR, international normalized ratio; AARP, AAR-platelet count score; P2/MS, monocyte fraction/segmented neutrophil fraction/platelet count index; PAPAS, Platelet count/age/ALP/AFP/AST index. 
Table II. Characteristics of patients with malignant hepatic tumors categorized according to recurrence status.

\begin{tabular}{|c|c|c|c|}
\hline Parameter & Recurrence & No recurrence & P-value \\
\hline Sex, male/female, $\mathrm{n}$ & $57 / 17$ & $88 / 37$ & 0.146 \\
\hline Age, years & $55 \pm 6$ & $56 \pm 11$ & 0.319 \\
\hline HBsAg, negative/positive, $n$ & $34 / 40$ & $62 / 53$ & 0.752 \\
\hline Ascites, no/yes, $\mathrm{n}$ & $63 / 11$ & $107 / 8$ & 0.608 \\
\hline Cirrhosis, no/yes, $\mathrm{n}$ & $39 / 35$ & $65 / 50$ & 0.808 \\
\hline Child-Pugh classification, $\mathrm{A} / \mathrm{B}, \mathrm{n}$ & $65 / 9$ & $93 / 22$ & 0.016 \\
\hline Diameter of spleen, mm (range) & $108.90(75.30-175.30)$ & $107.15(72.8-194.1)$ & 0.924 \\
\hline Polycythemia, no/yes, & $64 / 10$ & $111 / 4$ & 0.291 \\
\hline Non-surgical/surgical treatment, $\mathrm{n}$ & $35 / 39$ & $63 / 52$ & 0.232 \\
\hline Tumor size, $<5 / \geq 5 \mathrm{~cm}, \mathrm{n}$ (range) & $6(2.2-18)$ & $7(1.5-23)$ & 0.525 \\
\hline Tumor amount, single/multiple, $\mathrm{n}$ & $44 / 30$ & $86 / 29$ & 0.078 \\
\hline Vascular cancer embolus, no/yes & $55 / 19$ & $102 / 13$ & 0.074 \\
\hline $\mathrm{ALB}(\mathrm{ng} / \mathrm{ml})$ & $38.61 \pm 5.23$ & $37.16 \pm 5.78$ & 0.087 \\
\hline $\operatorname{ALT}(\mathrm{U} / \mathrm{l})$ & $41.7(7-212)$ & $41.50(11-661)$ & 0.949 \\
\hline AST (U/l) & $50(16-438)$ & $49(13-661)$ & 0.741 \\
\hline ALP (U/l) & $134.9(48-836)$ & $122.40(20-1,854.50)$ & 0.549 \\
\hline GGT (U/l) & $113.30(10-1,257)$ & $91.50(5-1,048)$ & 0.426 \\
\hline AFP, ng/ml (range) & $69.33(0.82-2,000)$ & $29.78(0.72-2,000)$ & 0.370 \\
\hline Total protein, g/l (range) & $69.60(50-91.7)$ & $68.55(31-97)$ & 0.173 \\
\hline PLT, $x 10^{9} / 1$ (range) & $137(50-537)$ & $135.5(37-454)$ & 0.931 \\
\hline PT (range) & $12.7(10-19.7)$ & $12.80(8.90-20.20)$ & 0.371 \\
\hline INR (range) & $1.06(0.84-1.67)$ & $1.07(0.75-1.74)$ & 0.370 \\
\hline Mononuclear cell, \% (range) & $7.24(1.2-18.81)$ & $7.39(3-23.51)$ & 0.438 \\
\hline Lymphocyte, \% (range) & $30.33 \pm 9.18$ & $27.16 \pm 10.83$ & 0.042 \\
\hline Neutrophil, \% (range) & $57.19 \pm 12.81$ & $61.96 \pm 13.07$ & 0.016 \\
\hline Cholesterol, mmol/l & $4.05 \pm 1.30$ & $3.71 \pm 0.96$ & 0.042 \\
\hline AARP, negative/positive, $\mathrm{n}$ & $17 / 57$ & $21 / 94$ & 0.985 \\
\hline Pohl score, negative/positive, $\mathrm{n}$ & $47 / 27$ & $74 / 41$ & 0.749 \\
\hline API (range) & $7(1-10)$ & $7(0-10)$ & 0.438 \\
\hline CDS (range) & $6(2-9)$ & $6(2-9)$ & 0.693 \\
\hline APRI (range) & $1.057(0.15-16.47)$ & $0.925(0.11-11.3)$ & 0.733 \\
\hline King's score (range) & $23.029(2.91-305.10)$ & $23.27(2.03-267.06)$ & 0.564 \\
\hline Lok index (range) & $0.58(0.06-0.98)$ & $0.54(0.02-0.99)$ & 0.515 \\
\hline $\mathrm{P} 2 / \mathrm{MS}$ (range) & $44.23(3.45-612.79)$ & $49.88(1.05-1,684.78)$ & 0.536 \\
\hline PAPAS (range) & $3.17(1.71-5.63)$ & $3.01(1.29-8.97)$ & 0.588 \\
\hline PSR (range) & $1.32(0.35-4.33)$ & $1.22(0.28-4.99)$ & 0.701 \\
\hline S-index (range) & $0.605(0.03-10.9)$ & $0.53(0.02-4.84)$ & 0.800 \\
\hline FIB-4 (range) & $2.71(0.5-24.5)$ & $2.88(0.49-26.59)$ & 0.556 \\
\hline FibroQ (range) & $4.21(0.91-22.11)$ & $4.88(1.15-45.83)$ & 0.583 \\
\hline GUCI (range) & $42.66(5.15-663.26)$ & $40.67(4.72-437.81)$ & 0.667 \\
\hline GPR (range) & $0.86(0.04-13.66)$ & $0.61(0.03-6.10)$ & 0.548 \\
\hline APGA (range) & $20.20(4.17-65.83)$ & $17.58(4.04-68.33)$ & 0.606 \\
\hline Forn index (range) & $9.75 \pm 2.09$ & $9.82 \pm 2.05$ & 0.835 \\
\hline PLR (range) & $123.69(31.60-588.64)$ & $97.65(18.67-589.29)$ & 0.001 \\
\hline
\end{tabular}

ALB, albumin; CEA, carcinoembryonic antigen; ALT, alanine aminotransferase; AST, aspartate aminotransferase; ALP, alkaline phosphatase; GGT, $\gamma$-glutamyl transpeptidase; AFP, $\alpha$-fetoprotein; PLT, platelet; API, age-PLT index; CDS, care dependency scale; APRI, AST to PLT ratio index; PSR, PLT count/spleen diameter ratio; FIB-4, fibrosis-4; GUCI, Göteborg University Cirrhosis Index; GRP, $\gamma$-glutamyl transpeptidase to PLT ratio; APGA, Aspartate aminotransferase/platelet count $/ \gamma$-glutamyl transpeptidase/alpha fetoprotein index; PLR, PLT to lymphocyte ratio; PT, Prothrombin time; INR, international normalized ratio; AARP, AAR-platelet count score; P2/MS, monocyte fraction/segmented neutrophil fraction/platelet count index; PAPAS, Platelet count/age/ALP/AFP/AST index. 
Table III. Ability of data to predict survival status of patients with malignant hepatic tumors.

\begin{tabular}{lcccc}
\hline Data & AUC & Cut-off & $95 \%$ CI & P-value \\
\hline AFP & 0.610 & 85.4 & $0.527-0.671$ & 0.0133 \\
Uric acid & 0.614 & 231 & $0.541-0.684$ & 0.0057 \\
Total protein & 0.585 & 71.9 & $0.511-0.656$ & 0.0429 \\
FIB-4 & 0.615 & 4.818 & $0.541-0.685$ & 0.0067 \\
FibroQ & 0.610 & 5.104 & $0.537-0.680$ & 0.0099 \\
Forns index & 0.595 & 11.059 & $0.522-0.666$ & 0.0258 \\
GPR & 0.591 & 0.869 & $0.518-0.662$ & 0.0297 \\
GUCI & 0.606 & 56.386 & $0.533-0.676$ & 0.0120 \\
King's score & 0.612 & 31.31 & $0.539-0.682$ & 0.0077 \\
Lok-index & 0.597 & 0.694 & $0.523-0.667$ & 0.0254 \\
P2/MS & 0.572 & 43.68 & $0.498-0.644$ & 0.0921 \\
PAPAS & 0.608 & 2.40 & $0.535-0.678$ & 0.0090 \\
PSR & 0.580 & 1.052 & $0.506-0.652$ & 0.0602 \\
S-index & 0.579 & 0.391 & $0.505-0.650$ & 0.0632 \\
APGA & 0.613 & 14.73 & $0.540-0.683$ & 0.0061 \\
API & 0.574 & 7 & $0.500-0.645$ & 0.0846 \\
APRI & 0.606 & 1.096 & $0.533-0.676$ & 0.0121 \\
CDS & 0.610 & 6 & $0.536-0.680$ & 0.0078 \\
PLR & 0.662 & 106 & $0.590-0.729$ & 0.0001 \\
\hline
\end{tabular}

AUC, area under the curve; CI, confidence interval; AFP, $\alpha$-fetoprotein; FIB-4, fibrosis-4; FibroQ, Fibro-quotient; GRP, $\gamma$-glutamyl transpeptidase to PLT ratio; GUCI, Goteburg University Cirrhosis Index; King's score, Fibrosis index based on the four factors; Lok-index, Fibrosis index based on the three factors; APGA, Aspartate aminotransferase/platelet count $/ \gamma$-glutamyl transpeptidase/alpha fetoprotein index; PSR, platelet count/spleen diameter $(\mathrm{mm})$ ratio index; S-index, $\gamma$-glutamyl transpeptidase/platelet count/serum albumin index; API, Age/platelet count index; PLR, PLT to lymphocyte ratio; PT, Prothrombin time; INR, international normalized ratio; APRI, Aspartate aminotransferase/platelet count ratio index; Forns index, Platelet count $/ \gamma$-glutamyl transpeptidase/age/cholesterol index; CDS, Cirrhosis discriminant score;P2/MS, monocyte fraction/ segmented neutrophil fraction/platelet count index; PAPAS, Platelet count/age/ALP/AFP/AST index.

Predictive indicators associated with mortality. Fig. 2A presents the overall survival rate of all patients. The results from a log-rank test demonstrated that age $>46$ years, vascular cancer embolus, AFP $>85.4 \mathrm{ng} / \mathrm{ml}$, ALP $>111 \mathrm{U} / \mathrm{ml}$, percentage of monocytes $>7$, percentage of neutrophils $>70.4$, total protein $>71.9 \mathrm{~g} / \mathrm{l}, \mathrm{HGB}>144 \mathrm{~g} / \mathrm{l}, \mathrm{RBC}>4.85 \times 10^{12} / 1$, monocyte $>7$ and uric acid $>231 \mu \mathrm{mol} / 1$ were significant predictors of a higher mortality rate (Table V). Among the 18 PLT-based models, APRI $>1.096$, FIB-4 $>4.818$, FibroQ $>5.104$, Forn index $>11.059$, GPR $>0.869$, GUCI $>56.386$, King's score $>31.31$, Lok-index $>0.695$, PAPAS $>2.405$, Pohl score (positive) and PLR $>106$ were significantly associated with a higher mortality rate (Table V). Fig. 3 presents the Kaplan-Meier curves of 12 PLT-based models (APRI, FIB-4, FibroQ, Forns index, GPR, GUCI, King's score, Lok index, PAPAS, Phol score, PLR and CDS) which were determined to be significant by ROC curve analysis. The higher scoring groups in the 18 PLT-based scoring
Table IV. Ability of data to predict recurrence status of patients with malignant hepatic tumors.

\begin{tabular}{lcccc}
\hline Data & AUC & Cut-off & $95 \%$ CI & P-value \\
\hline Uric acid & 0.583 & 325 & $0.509-0.654$ & 0.0482 \\
Lymphocyte & 0.597 & 22.4 & $0.523-0.667$ & 0.0191 \\
Neutrophil & 0.615 & 56.3 & $0.542-0.685$ & 0.0048 \\
FIB-4 & 0.515 & 4.817 & $0.442-0.589$ & 0.7186 \\
FibroQ & 0.525 & 7.833 & $0.451-0.598$ & 0.5608 \\
Forns index & 0.505 & 11.22 & $0.431-0.578$ & 0.9105 \\
GPR & 0.500 & 0.577 & $0.427-0.574$ & 0.9968 \\
GUCI & 0.511 & 33.806 & $0.437-0.584$ & 0.7994 \\
King's score & 0.508 & 28.397 & $0.435-0.582$ & 0.8430 \\
Lok index & 0.517 & 0.569 & $0.443-0.590$ & 0.6931 \\
P2/MS & 0.515 & 86.605 & $0.441-0.588$ & 0.7325 \\
PAPAS & 0.548 & 2.903 & $0.474-0.621$ & 0.2526 \\
APGA & 0.522 & 13.22 & $0.448-0.595$ & 0.6017 \\
APRI & 0.510 & 1.63 & $0.436-0.583$ & 0.8170 \\
PLR & 0.640 & 175 & $0.567-0.708$ & 0.0007 \\
\hline
\end{tabular}

AUC, area under the curve; CI, confidence interval. FIB-4, fibrosis-4; FibroQ, Fibro-quotient; GRP, $\gamma$-glutamyl transpeptidase to PLT ratio; GUCI, Goteburg University Cirrhosis Index; King's score, Fibrosis index based on the four factors; Lok-index, Fibrosis index based on the three factors; APGA, Aspartate aminotransferase/platelet count $/ \gamma$-glutamyl transpeptidase/alpha fetoprotein index; Forns index, Platelet count $/ \gamma$-glutamyl transpeptidase/age/cholesterol index; PLR, PLT to lymphocyte ratio; APRI, Aspartate aminotransferase/platelet count ratio index; P2/MS, monocyte fraction/segmented neutrophil fraction/platelet count index; PAPAS, Platelet count/age/ALP/AFP/AST index.

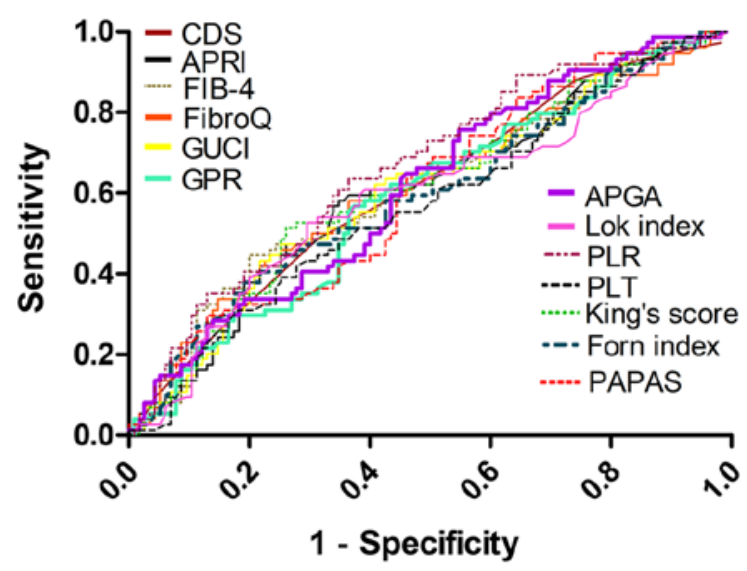

Figure 1. Receiver operating characteristic curves of 13 platelet-based models for predicting the risk of recurrence. The AUC of CDS was 0.610 $(\mathrm{P}=0.0078)$, the AUC of APRI was $0.606(\mathrm{P}=0.0121)$, the AUC of FIB-4 was $0.615(\mathrm{P}=0.0067)$, the AUC of FibroQ was $0.610(\mathrm{P}=0.0099)$, the AUC of GUCI was $0.606(\mathrm{P}=0.0120)$, the AUC of GPR was $0.591(\mathrm{P}=0.0297)$, the AUC of APGA was $0.613(\mathrm{P}=0.0061)$, the AUC of Lok index was 0.597 $(\mathrm{P}=0.0254)$, the AUC of PLR was $0.662(\mathrm{P}=0.0001)$, the AUC of PLT was $0.573(\mathrm{P}=0.089)$, the AUC of King's score was $0.612(\mathrm{P}=0.0077)$, the AUC of Forns index was $0.595(\mathrm{P}=0.0258)$ and the AUC of PAPAS was 0.608 $(\mathrm{P}=0.0090)$. AUC, area under the curve; CDS, care dependency scale; APRI, AST to PLT ratio index; FIB-4, fibrosis-4; GUCI, Göteborg University Cirrhosis Index; GPR, $\gamma$-glutamyl transpeptidase to PLT ratio; PLR, PLT to lymphocyte ratio; PLT, platelet; PAPAS, Platelet count/age/ALP/AFP/AST index; APGA, Aspartate aminotransferase/platelet count/GPR/AFP index. 
Table V. Predictors of mortality according to mortality time following log-rank test and multivariate analysis.

\begin{tabular}{|c|c|c|c|}
\hline \multirow[b]{2}{*}{ Variable } & \multirow{2}{*}{$\frac{\text { Log-rank test }}{\text { P-value }}$} & \multicolumn{2}{|c|}{ Multivariate analysis } \\
\hline & & HR (95\% CI) & P-value \\
\hline Age $>46$ years & 0.036 & $0.499(0.236-1.057)$ & 0.069 \\
\hline Cirrhosis & 0.06 & - & - \\
\hline HBV (positive) & 0.347 & - & - \\
\hline Ascites & 0.772 & - & - \\
\hline Multiple tumors & 0.059 & - & - \\
\hline Vascular cancer embolus & 0.001 & $0.520(0.287-0.943)$ & 0.031 \\
\hline Surgery & 0.488 & - & - \\
\hline $\mathrm{AFP}>85.4 \mathrm{ng} / \mathrm{ml}$ & 0.004 & $1.566(0.900-2.724)$ & 0.112 \\
\hline ALT $>32 \mathrm{U} / 1$ & 0.363 & - & - \\
\hline $\mathrm{AST}>34 \mathrm{U} / 1$ & 0.094 & - & - \\
\hline ALP $>111 \mathrm{U} / 1$ & 0.054 & - & - \\
\hline GGT >74 U/1 & 0.313 & - & - \\
\hline Total protein $>71.9 \mathrm{~g} / 1$ & 0.039 & $0.717(0.432-1.190)$ & 0.198 \\
\hline $\mathrm{PT}>13.3$ & 0.428 & - & - \\
\hline $\mathrm{HGB}>144 \mathrm{~g} / 1$ & 0.010 & $0.588(0.351-0.986)$ & 0.044 \\
\hline $\mathrm{RBC}>4.85 \times 10^{12} / 1$ & 0.040 & $0.903(0.459-1.775)$ & 0.767 \\
\hline Mononuclear cell $>7 \%$ & 0.022 & $0.740(0.420-1.303)$ & 0.297 \\
\hline Neutrophil > 70.4\% & 0.047 & $1.394(0.677-2.873)$ & 0.368 \\
\hline $\mathrm{PLT} \leq 113 \times 10^{9} / 1$ & 0.184 & - & - \\
\hline Uric acid $>231 \mu \mathrm{mol} / 1$ & 0.001 & $0.324(0.153-0.688)$ & 0.003 \\
\hline Tumor sizes $\geq 5 \mathrm{~cm}$ & 0.211 & - & - \\
\hline APGA > 14.733 & 0.117 & - & - \\
\hline $\mathrm{API}>7$ & 0.370 & - & - \\
\hline APRI > 1.096 & 0.012 & $2.323(0.790-6.827)$ & 0.126 \\
\hline $\mathrm{CDS}>6$ & 0.087 & - & - \\
\hline FIB-4 >4.818 & 0.002 & $0.732(0.273-1.961)$ & 0.535 \\
\hline FibroQ > 5.104 & 0.015 & $1.068(0.359-3.176)$ & 0.906 \\
\hline Forns_index $>11.059$ & 0.035 & $0.652(0.295-1.443)$ & 0.292 \\
\hline GPR $>0.869$ & 0.026 & $1.037(0.591-1.819)$ & 0.900 \\
\hline GUCI > 56.386 & 0.017 & $0.691(0.291-1.637)$ & 0.401 \\
\hline King's_score $>31.31$ & 0.004 & $0.854(0.279-2.612)$ & 0.781 \\
\hline Lok index $>0.695$ & 0.009 & $0.431(0.268-0.695)$ & 0.001 \\
\hline $\mathrm{P} 2 / \mathrm{MS} \leq 43.682$ & 0.053 & & \\
\hline PAPAS > 2.405 & 0.010 & $0.668(0.309-1.442)$ & 0.304 \\
\hline $\mathrm{PSR} \leq 1.056$ & 0.087 & - & - \\
\hline S-index $>0.391$ & 0.070 & - & - \\
\hline Pohl score (positive) & 0.028 & $0.834(0.395-1.758)$ & 0.633 \\
\hline AARP & 0.785 & - & - \\
\hline PLR > 106 & 0.001 & $0.862(0.561-1.307)$ & 0.090 \\
\hline
\end{tabular}

CI, confidence interval; HR, hazard ratio; ALT, alanine aminotransferase; AST, aspartate aminotransferase; ALP, alkaline phosphatase; GGT, $\gamma$-glutamyl transpeptidase; AFP, $\alpha$-fetoprotein; HGB, hemoglobin; RBC, red blood cell; PLT, platelet; API, age-PLT index; CDS, care dependency scale; APRI, AST to PLT ratio index; PSR, PLT count/spleen diameter ratio; FIB-4, fibrosis-4; GUCI, Göteborg University Cirrhosis Index; GRP, $\gamma$-glutamyl transpeptidase to PLT ratio; APGA, Aspartate aminotransferase/platelet count/ $\gamma$-glutamyl transpeptidase/alpha fetoprotein index; PLR, PLT to lymphocyte ratio; PT, Prothrombin time; INR, international normalized ratio; AARP, AAR-platelet count score; P2/MS, monocyte fraction/segmented neutrophil fraction/platelet count index; PAPAS, Platelet count/age/ALP/AFP/AST index; Lok-index, Fibrosis index based on the three factors; King's score, Fibrosis index based on the four factors; S-index, $\gamma$-glutamyl transpeptidase/platelet count/serum albumin index.

models had an increased risk of death compared with groups with lower scores. According to Cox multivariate analysis, the present study demonstrated that vascular cancer embolus [hazard ratio (HR), 0.520; 95\% CI, 0.287-0.943; $\mathrm{P}=0.031$ ], uric 

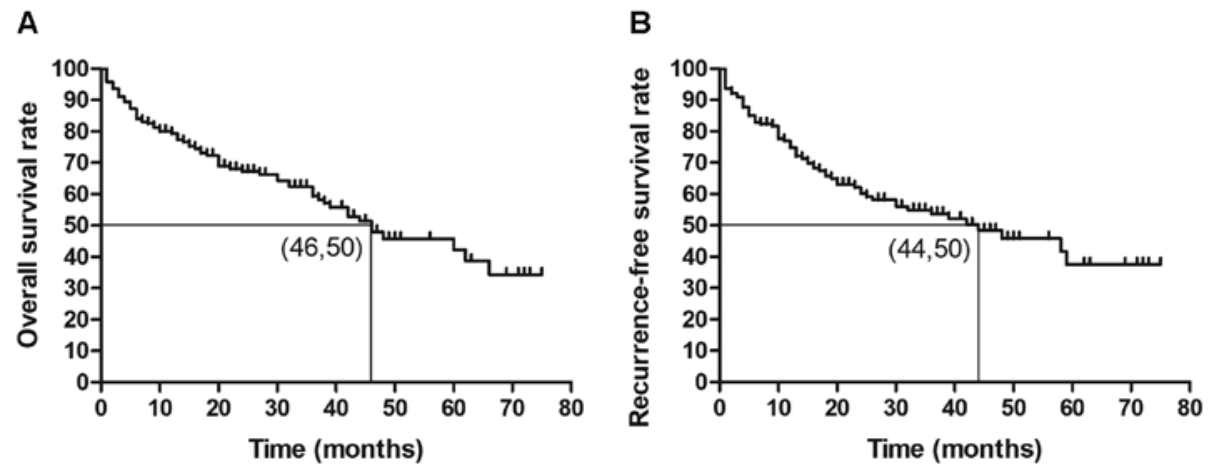

Figure 2. Overall survival and recurrence-free survival rates of patients with malignant hepatic tumors. (A) Kaplan-Meier survival curve of the estimated overall survival rate of 189 patients with malignant hepatic tumors; the median survival rate was 46.50 months. (B) Kaplan-Meier curve of the estimated recurrence-free survival rate of 189 patients with malignant hepatic tumors; the median recurrence-free survival rate was 44.50 months.
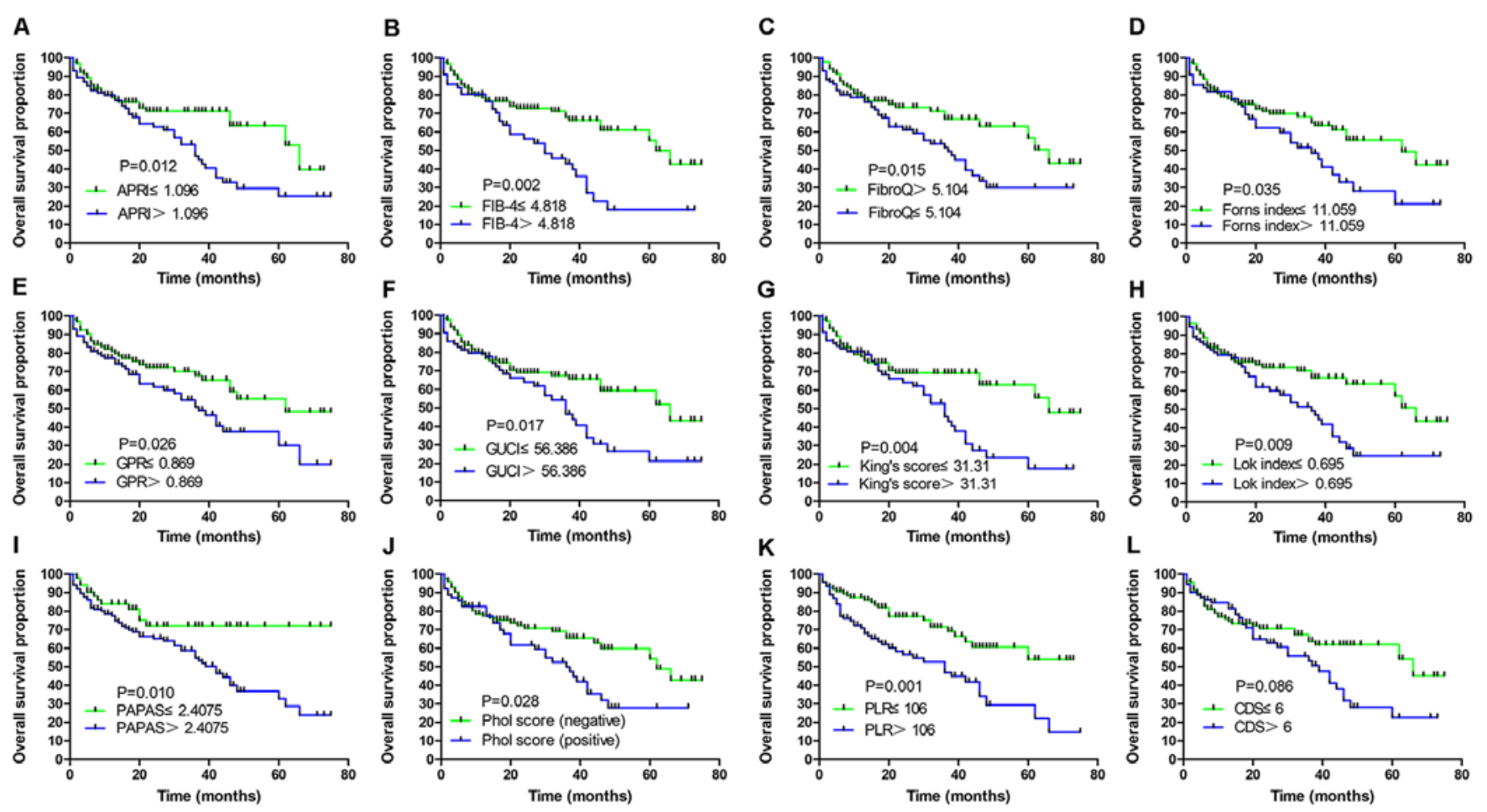

Figure 3. Overall survival rate of patients with malignant hepatic tumors. Kaplan-Meier curves of patients stratified according to (A) APRI, (B) FIB-4, (C) FibroQ, (D) Forns index, (E) GPR, (F) GUCI, (G) King's score, (H) Lok index, (I) PAPAS, (J) Phol score, (K) PLR and (L) CDS. CDS, care dependency scale; APRI, AST to PLT ratio index; FIB-4, fibrosis-4; GUCI, Göteborg University Cirrhosis Index; GPR, $\gamma$-glutamyl transpeptidase to PLT ratio; PLR, PLT to lymphocyte ratio; PLT, platelet; PAPAS, Platelet count/age/ALP/AFP/AST index; APGA, Aspartate aminotransferase/platelet count/ $\gamma$-glutamyl transpeptidase/AFP index.

acid $>231 \mu \mathrm{mol} / 1$ (HR, 0.324; 95\% CI, 0.153-0.688; P=0.003), HGB $>144 \mathrm{~g} / 1$ (HR, $0.588 ; 95 \% \mathrm{CI}, 0.351-0.986 ; \mathrm{P}=0.044)$ and Lok-index >0.695 (HR, 0.431; 95\% CI, 0.268-0.695; P=0.001) were independent risk factors of mortality (Table V).

Predictive indicators associated with recurrence. Fig. 2B presents the overall cumulative recurrence rate of all patients. In the present study, 74 patients presented with recurrence following surgery or non-surgical treatment, such as microwave ablation. The results from log-rank test demonstrated that the factors significantly associated with recurrence included the tumor volume, the presence of vascular cancer embolus, AFP $>85.4 \mathrm{ng} / \mathrm{ml}$, ALT $>32 \mathrm{U} / 1, \mathrm{HGB}>144 \mathrm{~g} / \mathrm{l}$, APRI $>1.01$, FIB-4 $>4.82$, King's score $>28.397$, PAPAS $>2.093$, Pohl score (positive) and PLR >175 (Table VI). The cumulative recurrence rates according to the PLT-based methods are presented in Fig. 4, the groups with higher scores had a greater risk of recurrence compared with groups with lower scores. The results from multivariate analysis demonstrated that vascular cancer embolus (HR, 0.427; 95\% CI, 0.237-0.770; $\mathrm{P}=0.005$ ), PLR >175 (HR, 0.302; 95\% CI, 0.183-0.498; P<0.001) and FIB-4 > 4.82 (HR, 0.447; 95\% CI, 0.232-0.607; P<0.001) were independent risk factors of recurrence (Table VI).

\section{Discussion}

Malignant hepatic tumors are the fourth leading cause of cancer-associated mortality worldwide (1), which is due to the 
A

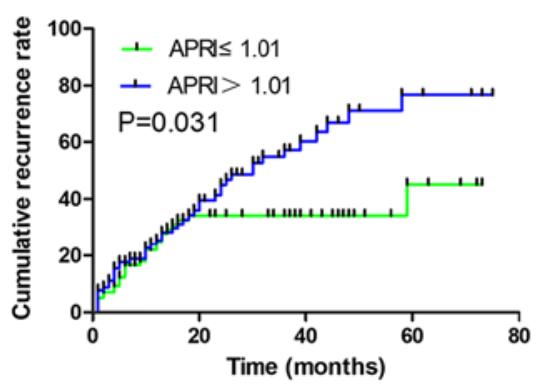

D

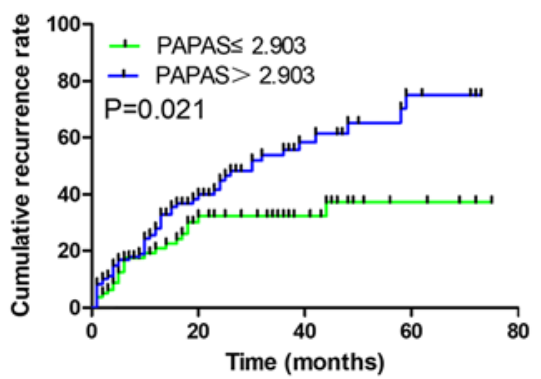

B

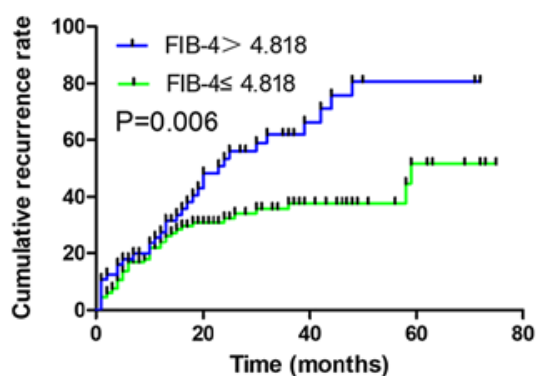

E

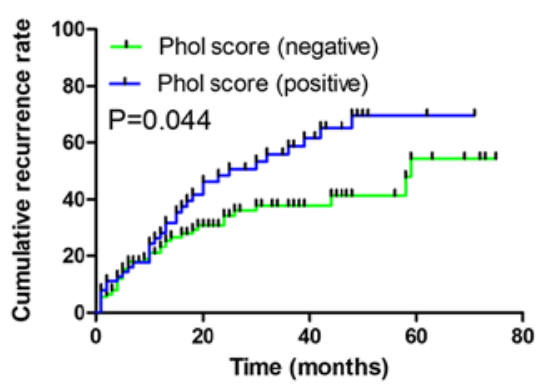

C

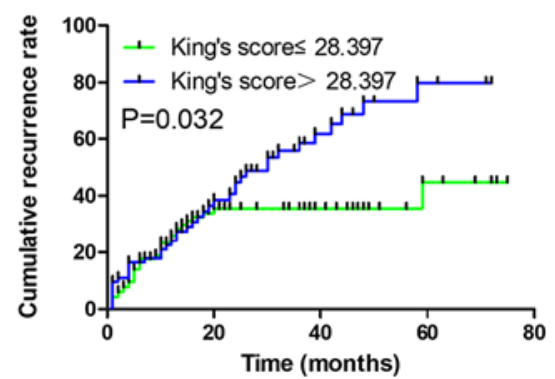

F

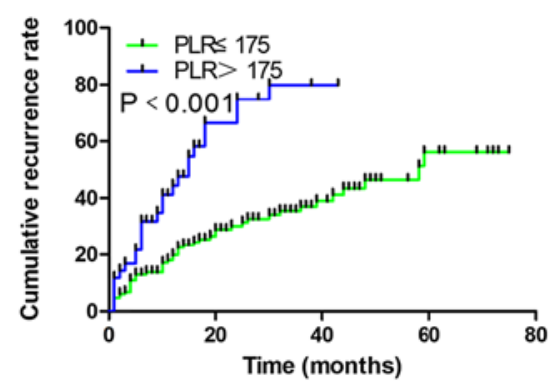

Figure 4. Cumulative recurrence rate of patients with malignant hepatic tumors. Kaplan-Meier curves of patients stratified according to (A) APRI, (B) FIB-4 index, (C) King's score, (D) PAPAS, (E) Phol score and (F) PLR. APRI, AST to PLT ratio index; FIB-4, fibrosis-4; PLR, platelet to lymphocyte ratio; PAPAS, Platelet count/age/ALP/AFP/AST index.

discreet early symptoms and the limited treatment options (56). Although the diagnosis and treatment of malignant hepatic tumors has been improved recently, $>50 \%$ patients are diagnosed in the middle and advanced stage and their prognosis is poor $(57,58)$. In addition, the 5-year recurrence probability following hepatectomy is 50-70\% (59-62). It is therefore crucial to identify risk factors for the prognosis of patients in order to prolong their survival time. The present study demonstrated that PLT-based models may serve a crucial role in the survival of patients. The results demonstrated that vessel carcinoma embolus, uric acid level, HGB level and Lok-index were independent predictors of overall survival of patients with malignant hepatic tumors. In addition, vessel carcinoma embolus, PLR and FIB-4 were independent risk factors of recurrence.

A previous study by $\mathrm{Du}$ et al (32) analyzed the prognostic value of PLT-based prognostic scores in patients with advanced malignant hepatic tumors who had received transarterial chemoembolization (TACE) therapy and reported that APGA is an independent risk factor for the overall survival rate. However, the present study determined the performance value of various scoring systems on the prognostic of patients with malignant hepatic tumors who received various types of therapy, including TACE and hepatectomy. In addition, only a small number of cases were included in previous studies and these studies only focused on the overall survival rate of patients $(25,32)$.

Numerous studies have reported that PLTs serve a crucial role in the occurrence and progression of liver tumors $(5,11,63)$. PLTs are involved in tumor growth and metabolism and vascular activation. Furthermore, tumor cells induce the activation and aggregation of PLTs through direct and indirect mechanisms, in order to achieve immune escape, tumor growth and tumor metastasis $(11,64)$. However, the association between PLT and the prognosis of patients with liver cancer remains controversial. A previous study demonstrated that the levels of PLT decreases before treatment, and that the overall risk and cancer-free mortality increased by 41 and $44 \%$ compared with patients with higher PLT levels, respectively (65). A lower PLT level presented a 0.67 -fold increase in the risk of overall mortality and a 0.44 -fold increase in the risk of disease-free death (the period after curative treatment when no disease can be detected) in comparison with a higher level of PLT in patients who underwent hepatectomy (65). A previous study demonstrated that decreased PLT levels were observed in patients treated with radiofrequency ablation, and that the risk of mortality in patients with low PLT level was $\sim 2 \mathrm{x}$ higher compared with patients with higher PLT levels (65). However, in the present study, PLT count was not significantly associated with postoperative survival rates.

The present study reported that Lok-index $>0.695$ was associated with poor overall survival following multivariate analysis, and that FIB-4 >4.82 and PLR >175 were associated with worse recurrence-free survival. Furthermore, higher scores indicated worse prognosis. The cut-off values corresponded to the maximal sum of sensitivity plus specificity. The cut-off values were therefore the best predictors of survival and recurrence status. Each PLT-based model corresponded to a cut-off value, and Kaplan-Meier survival curves and log-rank test were used to determine whether a value higher than the cut-off value predicted a high survival rate.

Previous studies have reported that PLT-based models can be used to predict patient survival (15,29-31). Similar to the present study, Qin et al (66) demonstrated that FIB-4 >3.25 is associated with a lower recurrence-free survival rate in patients with malignant hepatic tumors following surgery. Pang et al (24) reported that FIB-4 $>4.30$ is associated with a high recurrence risk and results from multivariate analysis 
Table VI. Predictors of recurrence stratified according to recurrence time following log-rank test and multivariate analysis.

\begin{tabular}{|c|c|c|c|}
\hline \multirow[b]{2}{*}{ Variable } & \multirow{2}{*}{$\frac{\text { Log-rank test }}{\text { P-value }}$} & \multicolumn{2}{|c|}{ Multivariate analysis } \\
\hline & & HR $(95 \% \mathrm{CI})$ & P-value \\
\hline Age $>63$ years & 0.619 & - & - \\
\hline Cirrhosis & 0.122 & - & - \\
\hline HBsAg & 0.566 & - & - \\
\hline Ascites & 0.526 & - & - \\
\hline Presence of $\geq 2$ tumors & 0.046 & $0.890(0.114-0.177)$ & 0.179 \\
\hline Vascular cancer embolus & 0.002 & $0.427(0.237-0.770)$ & 0.005 \\
\hline Surgery & 0.479 & - & - \\
\hline $\mathrm{AFP}>85.4 \mathrm{ng} / \mathrm{ml}$ & 0.002 & $1.169(0.719-1.902)$ & 0.529 \\
\hline $\mathrm{ALT}>32 \mathrm{U} / 1$ & 0.028 & $0.612(0.362-1.035)$ & 0.067 \\
\hline HGB > $144 \mathrm{~g} / 1$ & 0.006 & - & - \\
\hline $\mathrm{RBC}>4.91 \times 10^{12} / 1$ & 0.354 & - & - \\
\hline PLT $>113 \times 10^{9} / 1$ & 0.253 & - & - \\
\hline Uric acid $325 \mu \mathrm{mol} / 1$ & 0.077 & - & - \\
\hline Tumor size $>8.9 \mathrm{~cm}$ & 0.628 & - & - \\
\hline APGA > 17.72 & 0.133 & - & - \\
\hline $\mathrm{API}>2$ & 0.146 & - & - \\
\hline APRI >1.01 & 0.031 & $1.047(0.442-2.483)$ & 0.917 \\
\hline $\mathrm{CDS}>7$ & 0.317 & - & - \\
\hline FIB-4 >4.82 & 0.006 & $0.375(0.232-0.607)$ & $<0.001$ \\
\hline FibroQ $\leq 7.83$ & 0.115 & - & - \\
\hline Forns index $>11.22$ & 0.098 & - & - \\
\hline GPR $>0.577$ & 0.098 & - & - \\
\hline GUCI > 33.805 & 0.250 & - & - \\
\hline King's score $>28.397$ & 0.032 & $1.664(0.622-4.455)$ & 0.310 \\
\hline Lok index $>0.569$ & 0.146 & - & - \\
\hline $\mathrm{P} 2 / \mathrm{MS}>86.605$ & 0.922 & - & - \\
\hline PAPAS > 2.903 & 0.021 & $0.594(0.295-1.195)$ & 0.144 \\
\hline PSR $>1.828$ & 0.061 & - & - \\
\hline S-index >2.209 & 0.968 & - & - \\
\hline Pohl score (positive) & 0.044 & $0.664(0.371-1.190)$ & 0.169 \\
\hline AARP & 0.703 & - & - \\
\hline PLR > 175 & $<0.001$ & $0.302(0.183-0.498)$ & $<0.001$ \\
\hline
\end{tabular}

CI, confidence interval; HR, hazard ratio; ALT, alanine aminotransferase; AFP, $\alpha$-fetoprotein; HGB, hemoglobin; RBC, red blood cell; PLT, platelet; API, age-PLT index; CDS, care dependency scale; APRI, AST to PLT ratio index; PSR, PLT count/spleen diameter ratio; FIB-4, fibrosis-4; GUCI, Göteborg University Cirrhosis Index; GRP, $\gamma$-glutamyl transpeptidase to PLT ratio; APGA, Aspartate aminotransferase/platelet count $/ \gamma$-glutamyl transpeptidase/alpha fetoprotein index; PLR, PLT to lymphocyte ratio; PT, Prothrombin time; INR, international normalized ratio; AARP, AAR-platelet count score; P2/MS, monocyte fraction/segmented neutrophil fraction/platelet count index; PAPAS, Platelet count/age/ALP/AFP/AST index; Lok-index, Fibrosis index based on the three factors; King's score, Fibrosis index based on the four factors; S-index, $\gamma$-glutamyl transpeptidase/platelet count/serum albumin index.

revealed that FIB-4 is an independent indicator of relapse. In addition, the present study demonstrated that PLR $>175$ was an independent indicator of recurrence. Increasing evidence has reported that a systemic inflammatory response is a crucial parameter for determining the prognosis of patients with various types of cancer $(67,68)$. Cancer-associated inflammation recruits regulatory $T$ cells and activates chemokines, which are associated with tumor growth and metastasis. Both neutrophilia and thrombocytosis represent nonspecific responses to cancer-associated inflammation (69). A meta-analysis and systematic review by Zheng et al (54) revealed that increased PLR is associated with HCC recurrence. Furthermore, PLR has been reported to be an independent risk factor for predicting recurrence-free survival in patients with HCC (54). The present study aimed to determine the performance of 18 scoring systems in predicting the overall survival and recurrence-free survival rates in patients with malignant hepatic tumors. Among the 18 PLT-based models, only Lok-index was 
associated with the overall survival rate of patients. In addition, Pang et al (24) demonstrated that APGA and PAPAS are better predictors of postoperative recurrence in patients with hepatocellular carcinoma compared with AAR, APRI, FIB-4 and FibroQ. It is unclear which model is more valuable for predicting the overall survival and recurrence-free survival of patients with malignant hepatic tumor. Therefore, additional studies are required to determine the value of each PLT-based model for predicting overall and recurrence-free survival.

In the present study, malignant hepatic tumors were more common in males compared with females, and single lesions were more common than multiple lesions. The male to female was 145:44 and the single to multiple lesion ratio was 5:2. However, sex and lesion number were not associated with risk of recurrence or mortality. These findings were similar to results from cohort studies reporting that sex and lesion number do not predict recurrence or mortality in patients with malignant hepatic tumors $(24,25)$.

The prognosis of malignant hepatic tumors remains poor due to the high recurrence rate. It is therefore crucial to identify certain prognosis factors in liver cancer. AFP is the most widely used marker to determine the prognosis of hepatocellular carcinoma; however, its diagnostic value remains poor due to its low sensitivity and specificity (70). An effective intervention model is therefore needed to evaluate the prognosis of patients with hepatocellular carcinoma. By contrast, the Lok-index, PLR and FIB-4 models are more available compared with APF as the parameters of the PLT-based score models are easy to access and were assessed in all the hospitalized patients. Therefore, Lox-index, PLR and FIB-4 were better at determining prognosis compared with AFP in the present study.

The present study exhibited certain limitations. Firstly, only 189 patients were included in the current study. Secondly, the performance of 18 PLT-based models used to assess the recurrence or survival status were unsatisfactory. This may be due to the relatively small number of patients and the shorter follow-up period. Thirdly, $50 \%$ patients presented with HBV infection. It is crucial to assess the results taking HCV infection into account, however the number of patients presenting with an $\mathrm{HCV}$ infection was too small in the present study to assess. Although FIB-4 and Lok-index models were better predictors of cirrhosis, further investigation is required to evaluate their role in patients without cirrhosis. In addition, the parameters of PLR include lymphocytes, and lymphocytes are markers of inflammation. Currently, there are few studies assessing the effect of lymphocytes on the prognosis of malignant hepatic tumor, and thus there are still a lack of relevant inflammatory indicators for comparison.

To the best of our knowledge, the present study was the first to assess the role of 18 PLT-based models in the prediction of recurrence and survival rates of patients with malignant hepatic tumors. The findings from the present study may help surgeons to better evaluate the prognosis of patients with malignant hepatic tumors following surgery, as PLT-based models represent non-invasive, low-cost and computable prognostic model which can be easily used.

In conclusion, the present study demonstrated that the Lok-index is a valuable predictor for the overall survival rate of patients with malignant hepatic tumors. FIB-4 and PLR models were also valuable factors for the prediction of recurrence-free survival rate in patients with malignant hepatic tumors.

\section{Acknowledgements}

The authors would like to thank Dr Min Chen (Eye, Ear, Nose and Throat Hospital of Fudan University, Shanghai, China) for her guidance on the design of the study.

\section{Funding}

The present study was supported by the National Key R\&D Program of China (grant no. 2017YFC0909900), the Clinical Medical Research Center of Echinococcosis in Qinghai Province (grant no. 2017-SF-L2) and the Health and Family Planning Commission Guiding Key Projects in Qinghai Province (grant no. 2016-wjzd-04).

\section{Availability of data and materials}

The datasets used and/or analyzed during the present study are available from the corresponding author on reasonable request.

\section{Authors' contributions}

CLH and QCD conceived and designed the study, collected and analyzed the data, and wrote and revised the manuscript. ZXW conceived and designed the study, collected and analyzed the data and wrote the manuscript. MQP, YYW and YYL participated in drafting and revising the manuscript, and collected the data. YZ revised the manuscript, and participated in the acquisition, analysis and interpretation of data. HJW and HNF designed the study, revised the manuscript and analyzed the data. All authors approved the final version of the manuscript.

\section{Ethics approval and consent to participate}

This study was approved by the Institutional Research Ethics Board of Qinghai University Affiliated Hospital (Xining, China) and followed the Declaration of Helsinki.

\section{Patient consent for publication}

Not applicable.

\section{Competing interests}

The authors declare that they have no competing interests.

\section{References}

1. Bray F, Ferlay J, Soerjomataram I, Siegel RL, Torre LA and Jemal A: Global cancer statistics 2018: GLOBOCAN estimates of incidence and mortality worldwide for 36 cancers in 185 countries. CA Cancer J Clin 68: 394-424, 2018.

2. Kew MC: Hepatocellular carcinoma: Epidemiology and risk factors. J Hepatocell Carcinoma 1: 115-125, 2014.

3. Buonaguro L, Petrizzo A, Tagliamonte M, Tornesello ML and Buonaguro FM: Challenges in cancer vaccine development for hepatocellular carcinoma. J Hepatol 59: 897-903, 2013. 
4. Kondo R, Nakashima O, Tanikawa K, Nomura Y and Kage M: Accumulation of platelets in the liver may be an important contributory factor to thrombocytopenia and liver fibrosis in chronic hepatitis C. J Gastroenterol 48: 526-534, 2013.

5. Li N: Platelets in cancer metastasis: To help the 'villain' to do evil. Int J Cancer 138: 2078-2087, 2016.

6. Zhang LQ, Xu XS, Wan Y, Song SD, Wang RT, Chen W, Wang ZX, Chang HL, Wei JC, Dong YF and Liu C: Prognostic implications of estrogen receptor 1 and vascular endothelial growth factor A expression in primary gallbladder carcinoma. World J Gastroenterol 21: 1243-1250, 2015.

7. Sullivan BH Jr and Tumen HJ: The effect of portacaval shunt on thrombocytopenia associated with portal hypertension. Ann Intern Med 55: 598-603, 1961

8. Felix WR Jr, Myerson RM, Sigel B, Perrin EB and Jackson FC: The effect of portacaval shunt on hypersplenism. Surg Gynecol Obstet 139: 899-904, 1974.

9. Hoffman R, Haim N and Brenner B: Cancer and thrombosis revisited. Blood Rev 15: 61-67, 2001

10. Donati MB and Falanga A: Pathogenetic mechanisms of thrombosis in malignancy. Acta Haematol 106: 18-24, 2001.

11. Guo F, Zhu X and Qin X: Platelet distribution width in hepatocellular carcinoma. Med Sci Monit 24: 2518-2523, 2018.

12. Labelle M, Begum S and Hynes RO: Direct signaling between platelets and cancer cells induces an epithelial-mesenchymal-like transition and promotes metastasis. Cancer Cell 20: 576-590, 2011.

13. Bambace NM and Holmes CE: The platelet contribution to cancer progression. J Thromb Haemost 9: 237-249, 2011.

14. Oft M, Peli J, Rudaz C, Schwarz H, Beug H and Reichmann E: TGF-beta1 and Ha-Ras collaborate in modulating the phenotypic plasticity and invasiveness of epithelial tumor cells. Genes Dev 10: 2462-77, 1996.

15. Pang Q, Qu K, Bi JB, Liu SS, Zhang JY, Song SD, Lin T, Xu XS, Wan Y, Tai MH, et al: Thrombocytopenia for prediction of hepatocellularcarcinoma recurrence: Systematic review and meta-analysis. World J Gastroenterol 21: 7895-7906, 2015.

16. Wai CT, Greenson JK, Fontana RJ, Kalbfleisch JD, Marrero JA, Conjeevaram HS and Lok SF: A simple noninvasive index can predict both significant fibrosis and cirrhosis in patients with chronic hepatitis C. Hepatology 38: 518-526, 2010.

17. Sterling RK, Lissen E, Clumeck N, Sola R, Correa MC, Montaner J, S Sulkowski M, Torriani FJ, Dieterich DT, Thomas DL, et al: Development of a simple noninvasive index to predict significant fibrosis in patients with $\mathrm{HIV} / \mathrm{HCV}$ coinfection. Hepatology 43: 1317-1325, 2010.

18. Pohl A, Behling C, Oliver D, Kilani M, Monson $\mathrm{P}$ and Hassanein T: Serum aminotransferase levels and platelet counts as predictors of degree of fibrosis in chronic hepatitis $\mathrm{C}$ virus infection. Am J Gastroenterol 96: 3142-3146, 2001

19. Hsieh YY, Tung SY, Lee IL, Lee K, Shen CH, Wei KL, Chang TS, Chuang CS, Wu CS and Lin YH: FibroQ: An easy and useful noninvasive test for predicting liver fibrosis in patients with chronic viral hepatitis. Chang Gung Med J 32: 614-622, 2009

20. Lok AS, Ghany MG, Goodman ZD, Wright EC, Everson GT, Sterling RK, Everhart JE, Lindsay KL, Bonkovsky HL, Di Bisceglie AM, et al: Predicting cirrhosis in patients with hepatitis $\mathrm{C}$ based on standard laboratoiy tests: Results of the HALT-C cohort. Hepatology 42: 282-292, 2005.

21. Wang HW, Peng CY, Lai HC, Su WP, Lin CH, Chuang PH, Chen SH, Chen $\mathrm{CH}$, Hsu WF and Huang GT: New noninvasive index for predicting liver fibrosis in Asian patients with chronic viral hepatitis. Sci Rep 7: 3259, 2017.

22. Bonacini M, Hadi G, Govindarajan S and Lindsay KL: Utility of a discriminant score for diagnosing advanced fibrosis or cirrhosis in patients with chronic hepatitis $\mathrm{C}$ virus infection. Am J Gastroenterol 92: 1302-1304, 1997.

23. Udell JA, Wang CS, Tinmouth J, Fitzgerald JM, Ayas NT, Simel DL, Schulzer M, Mak E and Yoshida EM: Does this patient with liver disease have cirrhosis? JAMA 307: 832-842, 2012.

24. Pang Q, Zhang JY, Xu XS, Song SD, Qu K, Chen W, Zhou YY, Miao RC, Liu SS, Dong YF and Liu C: Significance of platelet count and platelet-based models for hepatocellular carcinoma recurrence. World J Gastroenterol 21: 5607-5621, 2015.

25. Ho SY, Liu PH, Hsu CY, Chiou YY, Su CW, Lee YH, Huang YH, Lee FY, Hou MC and Huo TI: Prognostic performance of ten liver function models in patients with hepatocellular carcinoma undergoing radiofrequency ablation. Sci Rep 8: 843, 2018.
26. Chung HA, Kim JH, Hwang Y, Hong SC, Ko SY, Choe WH and Kwon SY: Noninvasive fibrosis marker can predict recurrence of hepatocellular carcinoma after radiofrequency ablation. Saudi J Gastroenterol 22: 57-63, 2016.

27. Han S, Lee S, Yang JD, Leise MD, Ahn JH, Kim S, Jung K, Gwak MS, Kim GS and Ko JS: Risk of posttransplant hepatocellular carcinoma recurrence is greater in recipients with higher platelet counts in living donor liver transplantation. Liver Transpl 24: 44-55, 2018

28. Gu L, Wen W, Wu Z, Bai K, Liu W, Lai G and Li D: Abnormal platelet count correlates with poor survival in hepatocellular carcinoma. Infection International 6: 93-102, 2018.

29. Metussin A, Patanwala I and Cross TJ: Partial hepatectomy vs. transcatheter arterial chemoembolization for resectable multiple hepatocellular carcinoma beyond Milan Criteria: A RCT. J Hepatol 62: 747-748, 2015.

30. Pang Q, Liu C, Zhang JY, Qu K, Song SD, Liu SS and Xu XS: Serotonin in liver tumor: Friend or foe? Hepatology 62: 319, 2015.

31. Pang Q, Liu C, Qu K, Liu S and Berasain C: Conflicting relationship between platelets and prognosis of hepatocellular carcinoma: Is platelet-derived serotonin involved in? Liver Int 35: 2484, 2015.

32. Du QC, Hu CL, Wang YY and Zhou Y: Comparison of the prognostic value of platelet-based prognostic models in patients with malignant hepatic tumors after TACE therapy. Acta Cir Bras 34: e201900710, 2019.

33. Yoon JH, Park JW and Lee JM: Noninvasive diagnosis of hepatocellular carcinoma: Elaboration on Korean liver cancer study group-national cancer center Korea practice guidelines compared with other guidelines and remaining issues. Korean J Radiol 17: 7-24, 2016.

34. Heimbach JK, Kulik LM, Finn RS, Sirlin CB, Abecassis MM, Roberts LR, Zhu AX, Murad MH and Marrero JA: AASLD guidelines for the treatment of hepatocellular carcinoma. Hepatology 67: 358-380, 2018.

35. Marrero JA, Ahn J and Rajender Reddy K; Americal College of Gastroenterology: ACG clinical guideline: The diagnosis and management of focal liver lesions. Am J Gastroenterol 109: $1328-1347,2014$

36. Korean Society of Abdominal Radiology: Diagnosis of hepatocellular carcinoma with gadoxetic acid-enhanced MRI: 2016 consensus recommendations of the Korean Society of abdominal radiology. Korean J Radiol 18: 427-443, 2017.

37. Paradis V and Bedossa $P$ : In the new area of noninvasive markers of hepatocellular carcinoma. J Hepatol 46: 9-11, 2007.

38. Gustafsson BI, Friman S, Mjornstedt L, Olausson $M$ and Backman L: Liver transplantation for polycystic liver diseaseindication and outcome. Transplant Proc 35: 813-814, 2003.

39. Gosalia AJ, Martin P and Jones PD: Advances and future directions in the treatment of hepatocellular carcinoma. Gastroenterol Hepatol (NY) 13: 398-410, 2017.

40. Bruix J, Gores GJ and Mazzaferro V: Hepatocellular carcinoma: Clinical frontiers and perspectives. Gut 63: 844-855, 2014.

41. Graf D, Vallböhmer D, Knoefel WT, Kröpil P, Antoch G, Sagir A and Häussinger D: Multimodal treatment of hepatocellular carcinoma. Eur J Intern Med 25: 430-7, 2014.

42. Child CG and Turcotte JG: Surgery and portal hypertension. In: Child CG, editor. The liver and portal hypertension. Philadelphia: WB Saunders pp50-72, 1964

43. Lee IC, Chan CC, Huang YH, Huo TI, Chu CJ, Lai CR, Lee PC, $\mathrm{Su} \mathrm{CW}$, Hung $\mathrm{HH}$, Wu JC, et al: Comparative analysis of noninvasive models to predict early liver fibrosis in hepatitis B e Antigen-negative Chronic Hepatitis B. J Clin Gastroenterol 45: 278-285, 2011.

44. Poynard T and Bedossa P: Age and platelet count: A simple index for predicting the presence of histological lesions in patients with antibodies to hepatitis $C$ virus. METAVIR and CLINIVIR Cooperative Study Groups. J Viral Hepat 4: 199-208, 2010

45. Islam S, Antonsson L, Westin J and Lagging M: Cirrhosis in hepatitis $C$ virus- infected patients can be excluded using an index of standard biochemical serum markers. Scand J Gastroenterol 40: 867-872, 2005 .

46. Cross TJ, Rizzi P, Berry PA, Bruce M, Portmann B and Harrison PM: King's Score: An accurate marker of cirrhosis in chronic hepatitis C. Eur J Gastroenterol Hepatol 21: 730-738, 2009.

47. Park YE, Kim BK, Park JY, Kim DY, Ahn SH, Han KH, Han S, Jeon MY, Heo JY and Song K: Gamma-glutamyl transpeptidase-to-platelet ratio is an independent predictor of hepatitis B virus-related liver cancer. J Gastroenterol Hepatol 64 (Suppl): S343, 2016 
48. Zhou K, Gao CF, Zhao YP, Liu HL, Zheng RD, Xian JC, Xu HT, Mao YM, Zeng MD and Lu LG: Simpler score of routine laboratory tests predicts liver fibrosis in patients with chronic hepatitis B. J Gastroenterol Hepatol 25: 1569-1577, 2010.

49. Güzelbulut F, Çetınkaya ZA, Sezıklı M, Yaşar B, Ozkara S and Övünç AO: AST-platelet ratio index, Forns index and FIB-4 in the prediction of significant fibrosis and cirrhosis in patients with chronic hepatitis C. Turk J Gastroenterol 22: 279-285, 2011.

50. Seto WK, Lee CF, Lai CL, Ip PP, Fong DY, Fung J, Wong DK and Yuen MF: A new model using routinely available clinical parameters to predict significant liver fibrosis in chronic hepatitis B. PLoS One 6: e23077, 2011.

51. Fung J, Lai CL, Fong DY, Yuen JC, Wong DK and Yuen MF: Correlation of liver biochemistry with liver stiffness in chronic hepatitis B and development of a predictive model for liver fibrosis. Liver Int 28: 1408-1416, 2010.

52. Lee JH, Yoon JH, Lee CH, Myung SJ, Keam B, Kim BH, Chung GE, Kim W, Kim YJ, Jang JJ and Lee HS: Complete blood count reflects the degree of oesophageal varices and liver fibrosis in virus-related chronic liver disease patients. J Viral Hepat 16: 444-452, 2009.

53. Testa R, Testa E, Giannini E, Borro P, Milazzo S, Isola L, Ceppa P, Lantieri PB and Risso D: Noninvasive ratio indexes to evaluate fibrosis staging in chronic hepatitis C: Role of platelet count/spleen diameter ratio index. J Intern Med 260: 142-150, 2010.

54. Zheng J, Cai J, Li H, Zeng K, He L, Fu H, Zhang J, Chen L, Yao J, Zhang Y and Yang Y: Neutrophil to lymphocyte ratio and platelet to lymphocyte ratio as prognostic predictors for hepatocellular carcinoma patients with various treatments: A meta-analysis and systematic review. Cell Physiol Biochem 44: 967-981, 2017.

55. Labori KJ, Guren MG, Brudvik KW, Røsok BI, Waage A, Nesbakken A, Larsen S, Dueland S, Edwin B and Bjørnbeth BA: Resection of synchronous liver metastases between radiotherapy and definitive surgery for locally advanced rectal cancer: Short term surgical outcomes, overall and recurrence free survivals. Colorectal Dis 19: 731-738, 2017.

56. Huang W, Skanderup AJ and Lee CG: Advances in genomic hepatocellular carcinoma research. Gigascience 7: gly135, 2018.

57. Hussain SA, Ferry DR, El-Gazzaz G, Mirza DF, James ND, McMaster P and Kerr DJ: Hepatocellular carcinoma. Ann Oncol 12: 161-172, 2001.

58. Zhong JH, Peng NF, You XM, Ma L, Xiang X, Wang YY, Gong WF, Wu FX, Xiang BD and Li LQ: Tumor stage and primary treatment of hepatocellular carcinoma at a large tertiary hospital in China: A real-world study. Oncotarget 8: 18296-18302, 2017.

59. Imamura H, Tanaka E, Matsuyama Y, Miyagawa S I, Kawasaki S, Kubota K, Takayama $\mathrm{T}$ and Makuuchi M: Risk factors contributing to early and late phases of intrahepatic recurrence of hepatocellular carcinoma (HCC) after hepatectomy. Gastroenterology 118: A916, 2000.
60. Minagawa M, Makuuchi M, Takayama T and Kokudo N: Selection criteria for repeat hepatectomy in patients with recurrent hepatocellular carcinoma. Ann Surg 238: 703-710, 2003

61. Zhang X, Li C, Wen T, Yan L, Li B, Yang J, Wang W, Xu M, Lu W and Jiang L: Appropriate treatment strategies for intrahepatic recurrence after curative resection of hepatocellular carcinoma initially within the Milan criteria: According to the recurrence pattern. Eur J Gastroenterol Hepatol 27: 933-940, 2015.

62. Hasegawa K, Kokudo N, Makuuchi M, Izumi N, Ichida T, Kudo M, Ku Y, Sakamoto M, Nakashima O, Matsui O and Matsuyama Y: Comparison of resection and ablation for hepatocellular carcinoma: A cohort study based on a Japanese nationwide survey. J Hepatol 58: 724-729, 2013.

63. He AD, Xie W, Song W, Ma YY, Liu G, Liang ML, Da XW, Yao GQ, Zhang BX, Gao CJ, et al: Platelet releasates promote the proliferation of hepatocellular carcinoma cells by suppressing the expression of KLF6. Sci Rep 7: 3989, 2017.

64. Wojtukiewicz MZ, Sierko E, Hempel D, Tucker SC and Honn KV: Platelets and cancer angiogenesis nexus. Cancer Metastasis Rev 36: 249-262, 2017.

65. Pang Q, Qu K, Zhang JY, Song SD, Liu SS, Tai MH, Liu HC and Liu C: The prognostic value of platelet count in patients with hepatocellular carcinoma. Medicine 94 (Baltimore): e1431, 2015.

66. Qin W, Wang L, Hu B, Leng S, Tian H, Luo H, Yao J, Chen X, Wu C, Chen G and Yang Y: A novel score predicts HBV-related hepatocellular carcinoma recurrence after hepatectomy: A retrospective multicenter study. J Gastrointest Surg 23: 922-932, 2018.

67. Mantovani A, Allavena P, Sica A and Balkwill F: Cancer-related inflammation. Nature 454: 436-444, 2008.

68. Balkwill $\mathrm{F}$ and Mantovani A: Inflammation and cancer: Back to Virchow? Lancet 357: 539-545, 2001.

69. Bhatti I, Peacock O, Lloyd G, Larvin M and Hall RI: Preoperative hematologic markers as independent predictors of prognosis in resected pancreatic ductal adenocarcinoma: Neutrophil-lymphocyte vs. plateletlymphocyte ratio. Am J Surg 200: 197-203, 2010.

70. Saffroy R, Pham P, Reffas M, Takka M, Lemoine A and Debuire B: New perspectives and strategy research biomarkers for hepatocellular carcinoma. Clin Chem Lab Med 45: 1169-1179, 2007.

This work is licensed under a Creative Commons Attribution-NonCommercial-NoDerivatives 4.0 International (CC BY-NC-ND 4.0) License. 